\title{
DESCRIPTION DU MÂLE DE SYPHACIA PETRUSEWICZI RAUSCHI
}

\author{
(Quentin, 1969)
}

\author{
par J.-C. Quentin et M.-C. Gran \\ Laboratoire de Zoologie (Vers), associé au C.N.R.S., Muséum national d'Histoire naturelle, \\ 43, rue Cuvier, F 75231 Paris Cedex 05. \\ et Laboratoire de Biologie, Faculté des Sciences et Techniques, Sfax, Tunisie.
}

L'étude des Oxyures du genre Syphacia parasites de Rongeurs de familles récentes (Cricétidés, Gerbillidés, Muridés, Microtidés) a montré que ce groupe réunit un éventail de petites lignées dispersées à partir d'un seul type morphologique ancestral (Quentin, 1971).

Dans une de ces lignées, huit espèces et sous-espèces ont été regroupées par Quentin et Kinsella, 1972. Il s'agit de S. frederici, S. vandenbrueli, S. petrusewiczi, $S$. petrusewiczi rauschi, $S$. sigmodoni, $S$. samoredini, $S$. peromysci, S. alata. Les ressemblances de ces espèces entre elles sont plus étroites qu'avec les autres espèces du genre Syphacia. Elles présentent notamment un plateau céphalique aminci latéralement et les femelles s'ornent de deux larges ailes cervicales qui s'impriment profondément dans la vésicule céphalique.

Dans ce groupe l'extension géographique des Oxyures, et leur passage chez de nouveaux hôtes entraîne une différenciation croissante des caractères. Ainsi, à partir des espèces $S$. frederici, $S$. vandenbrueli, parasites de Muridés (France et Belgique) dont la morphologie reste encore primitive, la spéciation se manifeste avec l'apparition de deirides chez $S$. petrusewiczi, parasite de Microtidés (Pologne, Alaska), par la dilatation de l'extrémité céphalique chez $S$. peromysci parasite d'Hesperomyinés (U.S.A.) et par l'élargissement des ailes latérales chez $S$. alata parasite d'Hesperomyiné (Amérique du Sud).

La spéciation est plus difficile à observer chez les mâles ; ceux-ci sont plus rares et leurs différences morphologiques sont plus discrètes en raison de leur petite taille. Cependant elle peut être suivie au niveau de l'ornementation de la pièce accessoire

Accepté le 19 octobre 1976. 

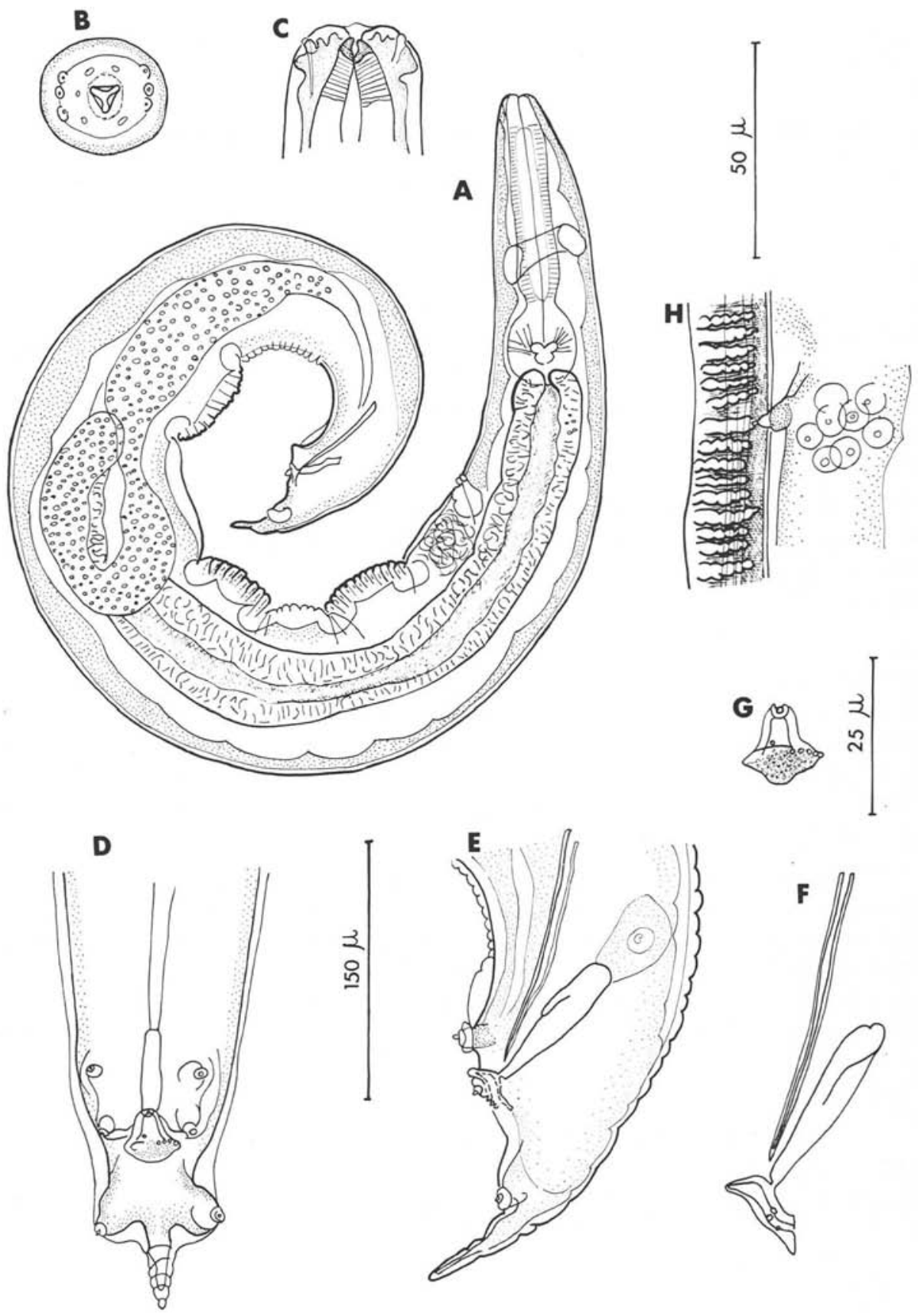
du gubernaculum. Cette ornementation est en effet absente chez les Syphacia parasites de Rongeurs paléarctiques, comme le montre la récente description de Jencev, 1973 en Bulgarie, du mâle de $S$. petrusewiczi. L'ornementation devient visible chez $S$. sigmodoni aux U.S.A.; elle est particulièrement différenciée chez $S$. peromysci et $S$. samarodini.

L'envoi par le $\mathrm{D}^{r}$ Rausch de l'Arctic Health Research de mâles et de femelles de Syphacia récoltés chez un Clethrionomys rutilus d'Alaska ( ${ }^{\circ} 40984$ localité : mile 20 Nabesna Rd; $\mathrm{n}^{\circ}$ MNHN 772 HA.) nous permet de compléter l'étude de cette lignée d'Oxyures par la description du mâle de Syphacia petrusewiczi rauschi, et de constater effectivement avec l'apparition d'une ornementation sur la pièce accessoire du gubernaculum, l'indice d'une spéciation.

\section{Description}

Ces spécimens appartiennent bien à la sous-espèce $S$. petrusewiczi rauschi comme nous avons pu le constater d'après les caractères céphaliques et cuticulaires de la femelle et d'après la forme des deirides pointues incluses dans chaque aile cervicale au niveau de l'anneau nerveux (fig. $1, H$ ).

Les mâles possèdent trois mamelons cuticulaires ventraux, un appendice caudal court, et une pièce accessoire au gubernaculum garnie de 3 à 4 bosses cuticulaires.

Les dimensions du spécimen représenté en A, B, C, D, E, F, G, et choisi pour type sont les suivantes :

Longueur : $1040 \mu$; largeur $95 \mu$; écart des pores amphidiaux $21 \mu$; anneau nerveux et pore excréteur situés respectivement à $90 \mu$ et $230 \mu$ de l'apex ; longueur totale de l'œsophage $170 \mu$; diamètre du bulbe $45 \times 40 \mu$. Le testicule naît à $645 \mu$ de l'apex et se replie sur lui-même à $525 \mu$ de l'apex au niveau du deuxième mamelon cuticulaire. Les trois mamelons cuticulaires débutent successivement à $280 \mu, 370 \mu$ et $500 \mu$ de l'apex et mesurent $54 \mu, 52 \mu$ et $60 \mu$. Dimensions du spicule : $56 \mu \times 3 \mu$; dimensions du gubernaculum sans son crochet : $35 \mu \times 8 \mu$. Dimensions du crochet accessoire : $15 \mu$ de long $\times 13 \mu$ de large. La disposition des papilles cloacales est représentée sur la figure $1, D$. La longueur de la queue est de $60 \mu$, celle de l'appendice caudal de $24 \mu$.

\section{Bibliographie}

Bernard (J.), 1966. - Nématodes de Micromammifères récoltés en Europe Centrale. Arch. Inst. Past. Tunis., 4, 609-632.

FIG. 1. - $\sigma^{*}$ de Syphacia petrusewiczi rauschi. A : $\sigma^{*}$ représenté en vue latérale; B et C: tête représentée en vue apicale et vue ventrale; D et E: bourse caudale représentée en vue ventrale et en vue latérale gauche; F : spicule et gubernaculum; G : crochet accessoire au gubernaculum en vue ventrale; $\mathrm{H}$ : deiride.

A : éch. $150 \mu ; \mathrm{B}, \mathrm{C}, \mathrm{D}, \mathrm{E}, \mathrm{H}$ : éch. $50 \mu ; \mathrm{G}$ et $\mathrm{F}$ : éch. $25 \mu$. 
JANČEV (J. I.), 1973. - New data on the taxonomy and distribution of Syphacia petrusewiczi Bernard, 1966. C.R. Acad. bulgare Sci., 26, 411-414.

QuENTIN (J.-C.), 1969. - Etude de Nématodes Syphacia parasites de Rongeurs Cricetidae sud-américains et de leurs corrélations biogéographiques avec certaines espèces néarctiques. Bull. Mus. nat. Hist. nat., 41, 909-925.

QueNTIN (J.-C.), 1971. - Morphologie comparée des structures céphaliques et génitales des Oxyures du genre Syphacia. Ann. Parasitol. hum. comp., 46, 15-60.

Quentin (J.-C.) et Kinsella (J.-M.), 1972. - Etude de trois espèces d'Oxyures Syphacia parasites de Rongeurs Cricétidés nord-américains. Hypothèses sur les filiations des espèces américaines. Ann. Parasitol. hum. comp., 47, $\mathrm{n}^{\circ}$ 5, 717-733. 